\title{
Exploração qualitativa do itinerário terapêutico de crianças com deficiência física na Nigéria usando análise de conteúdo temática
}

\author{
Qualitative exploration into therapeutic itinerary of children with physical disabilities in Nigeria using \\ thematic content analysis
} Exploración cualitativa del itinerario terapéutico de niños con discapacidades físicas en Nigeria
utilizando análisis de contenido temático

\author{
Chidozie Emmanuel Mbada ${ }^{1}$ [] \\ Adetola Taiwo Onilude ${ }^{1}$ (i) \\ Olufemi Oyeleye Oyewole ${ }^{2}$ (1) \\ Oluwatosin Eunice Olorunmoteni ${ }^{3}$ (1) \\ Clara Fatoye ${ }^{4}$ (1) \\ Abiola Oladele Ogundele ${ }^{5}$ (1) \\ Francis Fatoye ${ }^{4}$ (1)
}

1. Obafemi Awolowo University, Department of Medical Rehabilitation. lle-Ife, Nigeria.

2. Olabisi Onabanjo University Teaching Hospital, Department of Physiotherapy. Sagamu, Nigeria.

3. Obafemi Awolowo University, Department of Paediatrics and Child Health. Ile-Ife, Nigeria.

4. Manchester Metropolitan University. Faculty of Health, Department of Health Professions. Manchester, United Kingdom.

5. Bowen University, Department of Physiotherapy. Iwo, Nigeria.

\begin{abstract}
Resumo
Objetivo: Explorar o itinerário terapêutico na busca de cuidados para crianças com deficiências físicas pelos cuidadores. Métodos: Um plano qualitativo descritivo que recrutou nove cuidadores de crianças com deficiência física, com consentimento, que frequentavam um Hospital Universitário Nigeriano. Foi utilizado um guia de entrevista semi-estruturado para coletar dados sobre o itinerário terapêutico, que foi definido como os caminhos percorridos pelos indivíduos para resolver o seu problema de saúde. As entrevistas foram gravadas em áudio e transcritas literalmente. Os dados foram analisados utilizando a análise do conteúdo temático. Resultados: os temas emergentes revelaram que os cuidadores iniciaram o itinerário terapêutico para os respetivos filhos após a observação de quaisquer deficiências que vão para além de doenças casuais. A falta de capacidade dos hospitais privados, que servem como ponto inicial de entrada na busca de cuidados, encorajou a procura por cuidados nos hospitais públicos, lares espirituais, e clínicas tradicionais. A entrada na fisioterapia dependia de auto-referências, referências por parentes e médicos. Cuidar de crianças com deficiência física prejudicou de modo significativo a vida social, as finanças, o trabalho do cuidador; e expectativas não alcançadas de que a criança melhorasse o mais cedo possivel encorajaram um pluralismo contínuo. Conclusão e implicações para a prática: $O$ fato de ter crianças com deficiências e de ter havido atraso nos progressos levou os cuidadores a práticas múltiplas e complexas na busca de cuidados de saúde.
\end{abstract} Palavras-chave: Itinerário Terapêutico, Cuidadores; Crianças; Deficiências Físicas; Nigeria.

\begin{abstract}
Objective:To explore the therapeutic itinerary for seeking care for children with physical disabilities by caregivers. Methods: A descriptive qualitative design recruiting nine consenting caregivers of children with physical disabilities attending a Nigerian Teaching Hospital. A semi-structured interview guide was used to collect data on therapeutic itinerary which was defined as the paths taken by individuals to address their health problem. Interviews were audio-recorded and transcribed verbatim. Data was analyzed using thematic content analysis. Results: Emerging themes revealed that caregivers commenced therapeutic itinerary for their children upon observation of any impairments that go beyond casual illnesses. Lack of capacity in private hospitals, which serves as initial point of entry into care seeking, encourage vacillation into public hospitals, spiritual homes, and traditional clinics. Entry into physiotherapy was dependent on self-referrals, referrals by relatives, and physicians. Caring for children with physical disability significantly affected caregiver's social life, finances, work; and unmet expectations for a child to get well as soon as possible encouraged continuous pluralism. Conclusion and implications for the practice: Having children presenting with impairments and delayed milestones led to complex multiple health care seeking practices among caregivers. Thus, these caregivers become medical pluralists and covertly non-adherent to hospital treatment prescriptions.
\end{abstract}

Keywords: Therapeutic Itinerary; Caregivers; Children; Physical Disabilities; Nigeria.

\section{Resumen}

Objetivo: Explorar el itinerario terapéutico para la búsqueda de cuidado de niños con discapacidad física por parte de los cuidadores. Métodos: Un diseño cualitativo descriptivo que recluta a nueve cuidadores de niños con discapacidades físicas que asisten a un hospital universitario de Nigeria. Se utilizó una guía de entrevista semiestructurada para recopilar datos sobre el itinerario terapéutico que se definió como los caminos que recorren los individuos para abordar su problema de salud. Las entrevistas fueron grabadas en audio y transcritas textualmente. Los datos se analizaron mediante análisis de contenido temático. Resultados: Los temas emergentes revelaron que los cuidadores iniciaron un itinerario terapéutico para sus hijos al observar cualquier impedimento que vaya más allá de las enfermedades casuales. La falta de capacidad en los hospitales privados, que sirve como punto inicial de entrada en la búsqueda de atención, fomenta la vacilación en los hospitales públicos, hogares espirituales y clínicas tradicionales. La entrada en fisioterapia dependía de las autorremisiones, las derivaciones de familiares y médicos. El cuidado de niños con discapacidad física afectó significativamente la vida social, las finanzas y el trabajo del cuidador; y las expectativas no satisfechas de que un niño se recupere lo antes posible fomentaron el pluralismo continuo. Conclusión e implicaciones para la práctica: El hecho de que los niños presentaran discapacidades e hitos retrasados condujo a múltiples prácticas complejas de búsqueda de atención médica entre los cuidadores. Por lo tanto, estos cuidadores se vuelven médicos pluralistas y encubiertamente no se adhieren a las prescripciones de tratamiento hospitalario.

Palabras clave: Itinerario Terapéutico; Cuidadores; Niños; Discapacidades Físicas; Nigeria. 


\section{INTRODUÇÃO}

A carga Mundial de Morbilidade (Global Burden of Disease) analisou que $15 \%$ da população são moderadas ou são incapacitados, 93 milhões das pessoas que foram afetado sao crianças apartir de 14 anos, o nível das crianças com deficiência física são bem diferentes. ${ }^{1,2}$ As crianças com deficiência física são geralmente estimatizada na sociedade especialmente na África. ${ }^{3}$ Assim, a carga de viver como a criança incapacitada não só afeta as crianças mas também seus cuidadores. Porém, cuidar de uma criança com deficiência física é um acontecimento mais estressante que podem ocorrer. ${ }^{4}$ Particularmente, crianças com deficiência geralmente aumentar a vulnerabilidade dos seus pais ao estresse, isso que afeta mais as mães, cuidar de de criança incapacitadas pode ser físicalmente e mentalmente cansativo, por causa das demandas de cuidar deles 24 horas por dia e das suas necessidades complexas de cuidados de saúde..$^{5-8}$ Cuidadores das crianças incapacitadas são mais propensos a sofrer de depressão e angústia ${ }^{9}$ do que os cuidadores das crianças sem deficiência. ${ }^{9}$ No entanto, a quantidad ou magnitude e a natureza do desafio enfrentado pelos pais e cuidadores dependem da gravidade das deficiências físicas. ${ }^{10}$ Algumas das necessidades proeminentes relatadas por seus pais estão relacionadas a cuidados pessoais, ${ }^{11}$ gerenciamento de comportamentos adaptativos, ${ }^{12}$ médicos ${ }^{13}$ necessidades técnicas, financeiras e sociais. ${ }^{14}$ Assim, ter filhos com deficiência física representa uma sobrecarga significativa sobre os cuidadores para iniciar a busca de cuidados que, propositalmente ou inadvertidamente, os coloque em caminhos múltiplos e complexos.

Os caminhos percorrido pelos indivíduos para resolver seu problema de saúde são conhecido itinerário terapêutico. ${ }^{15}$ Muitas vezes as crianças com deficiência física e suas famílias precisar o intervenções de organização parapúblico como saúde, educação, serviço social ${ }^{16}$ e também as vezes buscam ajude fora da prática ortodoxa. Então para compreender o itinerário terapêutico dos cuidadores das crianças incapacitadas têm implicações políticas. No que diz respeito ao fornecimento de acesso accessível a cuidadores de saúde apoio multi- agências, apoio, apoio emocional e psicólogia e descanso para pais das crianças com deficiência ${ }^{17-19}$ Cuidar não é um evento estático ou um comportamento único, mas um processo dinâmico complexo que se desdobra ao longo do tempo e isso ressalta ainda mais as complexidades dinâmicas dos processos de prestação de cuidados. ${ }^{20}$ Estes exigem a exploração do itinerário terapêutico de crianças com deficiência para informar a melhoria na tomada de decisões políticas atendendo aos diversos aspectos de ajuda e apoio para essas crianças.

No entanto, foi informado que a cerca de $7 \%$ das na Nigéria crianças são observado e que tenham relatado deficiência neurológico, o sistema de saúde na Nigéria é fraca e fornece cuidado e apoio abaixo ideal para as crianças. ${ }^{21} \mathrm{Na}$ Nigéria, pais das crianças com deficiências então se procura tipos de terapia diferente aberta e veladas de saúde em buscar de restaurar o saúde das crianças deles. Eles seguem meio tradicional tradicionalista para as crianças com deficiência físicas pode ser informada pela probreza e esteriótipos baseada na cultura o que implicar que a essas tipos de crianças são sinais forma de punição de deuses e deus que torna os pais pluralistas médicos ou seja consulta várias fontes incluindo tradicionalista e lares espirituais na busca de ajuda para seus crianças com deficiência físicas ${ }^{22,23}$. Esse pluralismo médico pode atrasar o acesso aos cuidados de reabilitação e então o resultado podem ser abaixo do ideal. Então itinerário terapêutico dos pais e dos cuidadores de crianças incapacitadas precisa ser explorados para informar a tomada de decisão políticas. Infelizmente, esses ainda não está no perspectiva empírico. Portanto este estudo explorou itinerário terapêutico de buscar de cuidado a criança com deficiência física por cuidadores.

\section{MÉTODOS}

Este estudo qualitativo descritivo recrutou cuidadores consentidos de crianças com deficiência física atendidas no Ambulatório de Fisioterapia Infantil numa Hospital Universitário da Nigéria. Os entrevistados elegíveis eram pais/ responsáveis por crianças com deficiência física com idades entre 10 e 14 anos. Conforme ao sugerido de Creswell, ${ }^{24}$ 5-25 informantes são necessários para um estudo qualitativo. Portanto nove pais/ cuidadores foram envolvidos neste estudo. A localização em que o estudo foi realizado, as entrevistas foi baseado de uma experiência de primeiro contato com serviços de reabilitação incluindo fisioterapia não era comum. As crianças com deficiência física são aquelas por muito vezes precisam de serviços de reabilitação eles são referidos por um médico. A opinião pre-concebidos dos autores é de que criança mais velhas com deficiência tenham experiência substancial de itinerário terapêutico baseado na escolha de criança de 10 a 14 anos e moderada pelo formato de entrevista que permitiu um diálogo aberto os participantes de pesquisa.

Um roteiro de entrevista semi- estruturada foi utilizado para a coleção de dado sobre o itinerário terapêutico. O objetivo do estudo foi amplamente divulgado para os participantes, seus direitos de participação ou não é a garantia de proteção de suas identidades no armazenamento de dados e relatórios foram enfatizado. Posteriormente, o consentimento assinado foi obtido para participar do estudo. Além disso a permissão de gravar as entrevistas foi solicitada. Um dos autores (OAT) conduziu as entrevistas numa escritório livre cadê ruídos e cada entrevistas durou a cerca de 15 minutos. As entrevistas foram conduzidas em inglês ou lorubá, depende do idioma do melhor proficiência dos participantes. A língua iorubana é a língua indigena da Nigéria e ela é mais falada no cenário deste estudo. A tradução das entrevistas na língua loruba para o inglês foi feito por especialista do departamento de Linguística e Línguas Africana da Universidade da Nigéria.

As entrevistas gravadas em fita foram transcritas por palavra por palavra para preserva a originalidade códigos descritivos e curto foram alocado em seções do textos, e também códigos que expressam conceitos semelhantes foram agrupados para formar temas. A análise de conteúdo temática foi dedutiva de tema e frases- chave foi usada para organizar os dados e os narrações 
foram apresentada. ${ }^{25}$ Avaliação contemporâneo foi feito durante a análise para limitar os viesse potências associado á posição do autor entrevistador. Os temas foram revisados e auditados para garantir que estivessem fundamentados nas transcrições e bem representados nos dados com exemplos adequados, aumentando assim a confiabilidade do estudo. Uma trilha de auditoria também ajudou a reduzir o preconceito no estudo. Estatística descritiva de frequência, percentuais foram utilizados para resumir os dados sobre as variáveis sociodemográficas dos participantes e o resumo da análise qualitativa.

A aprovação ética do estudo foi obtida pelo Comitê de Ética e Pesquisa do Complexo Hospital Universitário Obafemi Awolowo (ERC / 2019/12/14).

\section{RESULTADOS}

As características demográfica dos participado mostram que mais da metade das mães três / mais filhos e procuram ajuda tardiamente (55.6\%) (Tabela 1). A Figura 1 mostra o caminho percorrido na busca pelo atendimento da criança com deficiência física. As mães expressaram vários caminhos na busca pelo cuidado de seus filhos com deficiência física. A Tabela 2 mostra a categoria, tema e subtema emergidos. Os resultados da análise qualitativa do estudo são apresentados em itálico.

\section{Fatores de risco para deficiência física}

A história da gravidez é uma das formas válidas de avaliar os fatores de risco pré-natal para comprometimento em crianças. Portanto, este estudo explorou a história do pré-natal das crianças com deficiência. Enquanto a maioria das mães relatou que suas gestações não foram associadas a nenhuma circunstância importante, outras relataram algumas incidências para as quais foram solicitadas intervenções. Uma mãe relatou:

Não houve muita coisa que rodeou a concepção dele, não passei mal durante a gravidez, aliás, eu faço checkup regular no hospital, o exame confirmava que o bebê estava bem no útero (P1).

Ao mesmo tempo, as incidências de indisposição durante a gravidez foram narradas por algumas das mães como capturadas por uma mãe:

Nem sabia que estava grávida porque ainda estava menstruada como naquela época, o médico depois confirmou que eu estava grávida de 2 meses, fiz o ultrassom da gravidez, ainda estava muito doente, minha sogra me deu algumas ervas, e eu estava bem (P8).

O estudo explorou lembranças de cuidadores sobre se a gravidez foi agitada ou não. Algumas mães retratam que suas gestações transcorreram sem intercorrências. Uma mãe respondeu:
Tabela 1. As características físicas entrevistados e resumo do registro de terapia infantil.

\begin{tabular}{|c|c|}
\hline $\begin{array}{c}\text { Variável } \\
\text { Socio-demograficas caraterísticas dos } \\
\text { entrevistado }\end{array}$ & $\mathrm{N}$ \\
\hline \multicolumn{2}{|l|}{ Estado civil } \\
\hline Casado & 9 \\
\hline \multicolumn{2}{|l|}{ Profissão } \\
\hline Professor & 2 \\
\hline Designer de moda & 1 \\
\hline Cozinheira & 3 \\
\hline Vendedora & 3 \\
\hline \multicolumn{2}{|l|}{ Número de filhos } \\
\hline Um & 2 \\
\hline Dois & 2 \\
\hline Três. & 2 \\
\hline Quatro & 3 \\
\hline \multicolumn{2}{|l|}{ Ambiente familiar } \\
\hline Monogâmico & 6 \\
\hline Polígamo & 3 \\
\hline \multicolumn{2}{|l|}{ Resumo do registro de terapia Infantil } \\
\hline \multicolumn{2}{|l|}{ Investigação Radiológica } \\
\hline Sim & 8 \\
\hline Não & 1 \\
\hline \multicolumn{2}{|l|}{ Check - up regulares } \\
\hline Sim & 8 \\
\hline Não & 1 \\
\hline \multicolumn{2}{|l|}{ Nascimento complicada } \\
\hline Sim & 6 \\
\hline Não & 3 \\
\hline \multicolumn{2}{|l|}{ Caraterísticas Perceptíveis da criança } \\
\hline Doente & 2 \\
\hline Atrasar marco de desenvolvimento & 5 \\
\hline Tudo acima & 2 \\
\hline \multicolumn{2}{|l|}{$\begin{array}{l}\text { Passo imediato dado quando a condição } \\
\text { foi notados }\end{array}$} \\
\hline Foi para o hospital & 2 \\
\hline Procurei conselho & 2 \\
\hline Procurei terapia alternative & 3 \\
\hline Tudo acima & 1 \\
\hline Nenhuma das acima & 1 \\
\hline \multicolumn{2}{|l|}{$\begin{array}{l}\text { Diferentes caminhos percorridos em } \\
\text { busca do cuidado }\end{array}$} \\
\hline Foi para o hospital & 3 \\
\hline Procurei terapia alternative & 1 \\
\hline Tudo acima & 5 \\
\hline \multicolumn{2}{|l|}{$\begin{array}{l}\text { Resposta imediata ao atendimento } \\
\text { hospitalar }\end{array}$} \\
\hline Cedo & 3 \\
\hline Tarde & 5 \\
\hline Não é específico & 1 \\
\hline
\end{tabular}




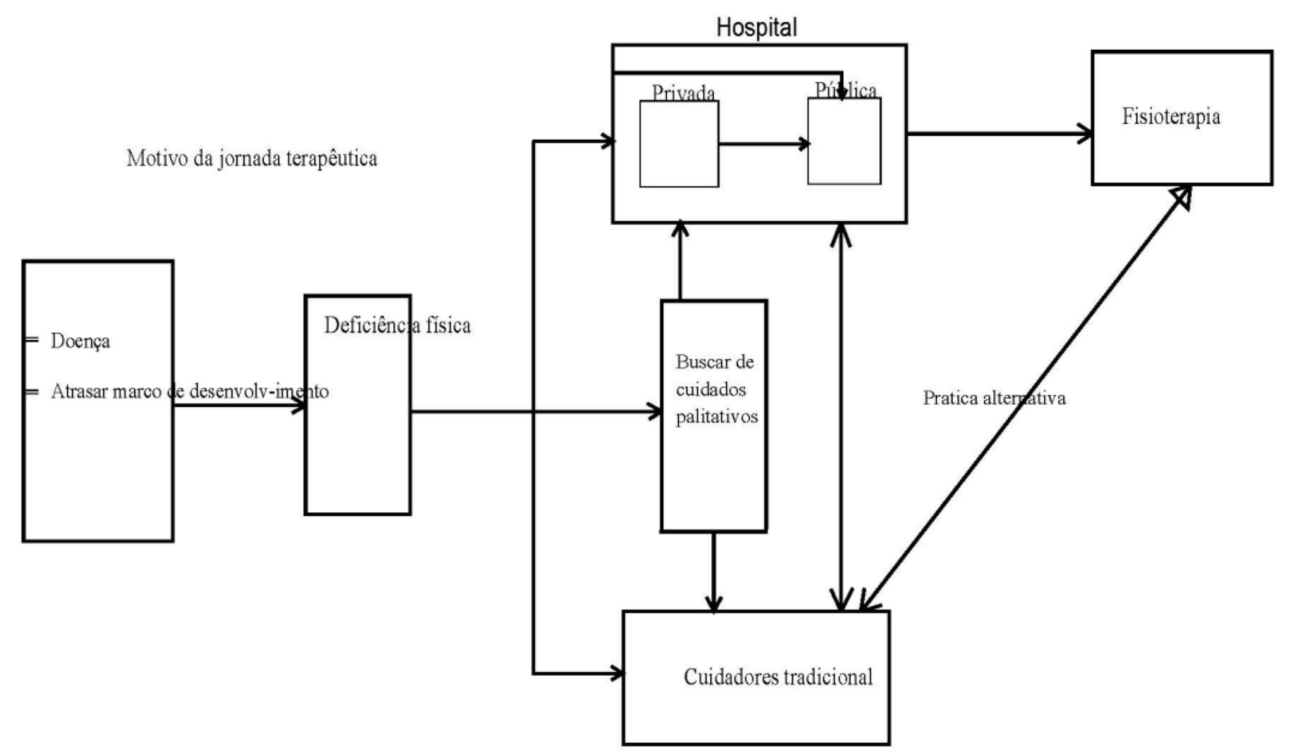

Figura 1. Caminho percorrido pelos respondentes do estudar do estudo ao buscar atendimento para crianças com deficiência físicas.

Tabela 2. Categoria, tema e subtema emergido do estudo.

\begin{tabular}{|c|c|c|}
\hline Categoria & Tema & Subtema \\
\hline \multirow{3}{*}{ Fatores de risco para deficiência física } & · História de gravidez & - Doença durante a gravidez \\
\hline & \multirow{2}{*}{ - Gravidez complicada } & - Trabalho de parto prolongado \\
\hline & & - Prematuro doença / doença neonatal \\
\hline \multirow{5}{*}{$\begin{array}{l}\text { Gatilhos de itinerário terapêutico } \\
\text { Ações terapêuticos }\end{array}$} & - Bem-estar da criança & - Doença estranha \\
\hline & - Impedimento e deficiência & - Paralisia do corpo \\
\hline & \multirow{3}{*}{ - Pluralismo de terapia } & - Consulta médica \\
\hline & & - Curandeiros tradicionais \\
\hline & & - Lares espirituais \\
\hline \multirow{3}{*}{ Experiência de reabilitação } & - Rota para fisioterapia. & $\begin{array}{l}\text { Referência médica e auto-referência } \\
\text { por meio de inquérito }\end{array}$ \\
\hline & \multirow[b]{2}{*}{ - Satisfatório e ajudavel } & - Menos tempo e espera \\
\hline & & $\begin{array}{l}\text { Consulta acessível e taxa de } \\
\text { tratamento. }\end{array}$ \\
\hline \multirow[b]{3}{*}{ Sobrecarga do itinerário terapêutico } & \multirow{3}{*}{ · Isolamento social/ emocional } & · Depressão \\
\hline & & - Perda de emprego \\
\hline & & - Baixa qualidade de vida \\
\hline
\end{tabular}


Eu dei à luz quando a gravidez estava com nove meses, e não foi por cesariana, mas por parto normal. Depois que eu dei a luz a ele, ele chorou sozinho, a enfermeira não batia antes dele chorar, posso dizer que ele estava forte e forte $(\mathrm{P} 1)$.

No entanto, outros relembraram suas experiências marcantes:

\section{Eu dei à luz em uma clínica tradicional após um trabalho} de parto prolongado de dois dias (P5).

Eu dei à luz por cesárea quando ele tinha 8 meses depois de um trabalho de parto prolongado de quase dois dias (P7).

Em suma, a maioria das mães passou por um trabalho de parto penoso e prolongado que variava de 8 horas a 3 dias. Além disso, partos prematuros foram mais relatados do que partos pós-termo. Nenhum dos casos de parto cesáreo foi eletivo, mas de emergência para salvar a mãe e / ou a criança.

\section{Gatilhos de itinerário terapêutico e ações terapêuticas}

O itinerário terapêutico das mães em relação aos filhos parece começar a partir das observações das mães sobre impedimentos e deficiências que vão além das doenças casuais que afetam os filhos. Normalmente, entre algumas mães, começou desde quando a criança era um recém-nascido até muitos meses após o parto. Os acionados foram variados conforme destacado:

não conseguia engatinhar, ficar em pé, ficava sempre curvado para frente, sem controle de pescoço (P4). quase todas as articulações do corpo dele estavam muito rígidas e ele não podia usar, era como se meu bebê já estivesse paralisado (P7).

As ações terapêuticas realizadas incluíram consultas com médicos, desde a Atenção Básica à Saúde até Hospital Universitário, curandeiros tradicionais e lares espirituais. Alguns informantes revelaram:

Eu o levei para uma matrona na minha rua. Eu o levei para o Centro de Saúde Primário. Posteriormente, foi encaminhado para esse serviço [Hospital Universitário], e posteriormente encaminhado para fisioterapia (P4).

Eu contei a minha mãe sobre isso, ela me levou a uma clínica tradicional de modelagem de ossos para tratadores levei ela pra orar na igreja, fiquei meses na igreja com ela (P9).

Isso também confirmou as práticas múltiplas das mães:
Os lugares que procuraram atendimento médico para ela incluem; igreja, hospital e teve uma época que minha irmã mais velha me levou a um curandeiro tradicional (P9).

Na tentativa de desvendar as tendências para o pluralismo médico existente entre as mães deste estudo, explorou-se o padrão de encaminhamento, o tempo de espera para as consultas iniciais e as barreiras para a busca por atendimento no Hospital Universitário onde este estudo foi realizado. Os resultados indicam que $o$ acesso direto ao cuidado, práticas de autorreferência e vacilações eram comuns. $\mathrm{O}$ acesso direto ao atendimento foi mais perceptível no patrocínio ao ambiente privado e ao Centro de Atenção Básica à Saúde. Um informante narrou:

Na verdade, quando aconteceu o incidente, levei ela correndo para um hospital particular (P3).

Normalmente, outras formas de práticas que envolvem a autorreferência e outras formas de encaminhamento, bem como a vacilação entre as diferentes modalidades de cuidado, geram-se após esses pontos de chamada iniciais. Ao afirmar as práticas de encaminhamento, os informantes afirmam:

O que aconteceu foi que eu já tinha levado ela no Hospital Geral antes de vir pra cá, vim sozinha (P2)

E em outros casos, os encaminhamentos vieram do primeiro ponto de chamada para o Hospital Universitário:

Ele foi encaminhado daquele hospital [Privado] para o Hospital Universitário para posterior tratamento (P3).

\section{Experiências de reabilitação}

As mães narraram suas experiências com a primeira $e$ subsequentes consultas dizendo:

Quando chegamos aqui, fui encaminhado primeiro para o pronto-socorro infantil e de lá ele foi encaminhado para a fisioterapia (P5).

As mães apreciam suas experiências como geralmente satisfatórias em termos de acessibilidade da taxa de consulta e tempo de espera razoável para consulta e tratamento:

Foi muito agradável porque atenderam ele na hora, o valor da consulta não era muito alto (P1).

De maneira geral, as mães afirmaram que suas visitas ao hospital têm sido úteis. Alguns dos trechos retratam esta visão:

Até agora tem ajudado porque tenho visto algumas mudanças na criança desde então tenho trazido para este hospital (P1). 
Tem sido útil ter visto várias mudanças e melhorias nela porque ela não conseguia se sentar bem antes (P2).

Foi mapeado o caminho da fisioterapia para a reabilitação das crianças. Na maioria dos casos, os médicos encaminhavam as mães para fisioterapia para as condições de seus filhos.

O médico foi quem o encaminhou para a fisioterapia (P4).

Outros fizeram auto-indagações e se referiram para fisioterapia:

Eu mesmo comecei a fisioterapia para ela, não fui encaminhada para cá, então vim para a fisioterapia por vontade própria (P2).

Para algumas das pacientes, a fisioterapia foi iniciada cedo, ao mesmo tempo que algumas dessas mães traziam seus filhos há um bom tempo.

Comecei cedo a fisioterapia para ele (P1).

E para alguns outros, a fisioterapia foi iniciada tarde devido a uma série de razões:

Comecei a fisioterapia para ela tarde por causa de dinheiro e algumas outras coisas pessoais (P9).

\section{Sobrecarga do itinerário terapêutico no cuidador e barreira na busca por atendimento}

Foi relatado que a procura de cuidados para crianças com deficiência física atrapalha a vida dos cuidadores, especialmente das mães. Exploramos como e em que medida o itinerário terapêutico dos cuidadores tem interferido em suas vidas. O exceto revela as posições de uma mãe:

O estado da criança atrapalhou tanto na família, .... não tem sido fácil, muitas vezes estou sempre deprimido, afetou muito a minha vida social, na maioria das vezes eu nem frequento eventos sociais, não tenho conseguido fazer quase tudo que eu deveria fazer, mas estou tentando fazer agora (P1).

O engajamento em práticas alternativas é freqüentemente influenciado por uma série de fatores que variam de fatores pessoais a pressões de colegas. Relações, amigos e pessoas de influência nortearam as decisões da maioria dos cuidadores.

Meu marido, amigos da família e as pessoas ao meu redor influenciaram algumas decisões que eu tomei no meu filho (P2).

A literatura é repleta de barreira á procura de atendimento em unidades públicas de saúde. Uma das consequências de tais barreiras é que engendra práticas alternativas. Os cuidadores narraram o grande revés da seguinte forma:

Finanças, restrições de tempo, restrições de trabalho / emprego, restrições de distância, questões de transporte e problemas sociais. Muitos contratempos e restrições, como restrições financeiras, restrições de tempo e um monte de coisas assim, estão impedindo os cuidados com o bebê... a distância é outro grande problema para mim, porque eu trago meu bebê desde muito cedo. distância só pra tratamento aqui (P4).

\section{DISCUSSÃO}

Este estudo explorou o itinerário terapêutico de busca de cuidado à criança com deficiência física por cuidadores. As crianças neste estudo eram heterogêneas em características, pois apresentam várias formas de deficiência. Normalmente, a literatura afirma que a deficiência em crianças frequentemente se apresenta com uma miríade de deficiências que podem envolver aspectos cognitivos, sensoriais, motores, sociais ou de comunicação / linguagem individualmente ou em combinação. ${ }^{26}$

Os bons cuidados durante a gravidez são importantes para a saúde da mãe e o desenvolvimento do feto. ${ }^{26} \mathrm{~A}$ gravidez é um momento crucial para promover comportamentos saudáveis e habilidades parentais. Um bom atendimento pré-natal vincula a mulher e sua família ao sistema formal de saúde, aumenta a chance de usar um atendente qualificado no parto e contribui para uma boa saúde ao longo do ciclo de vida. O cuidado inadequado durante esse período quebra uma ligação crítica no continuum do cuidado e afeta tanto as mulheres quanto os bebês. ${ }^{27} \mathrm{Um}$ terço de todas as mulheres grávidas neste estudo adoeceu durante a gravidez, algumas das quais exigiram hospitalização. Estudos têm demonstrado que a prevenção de problemas para mães e bebês depende de um continuum operacional de cuidados com cuidados acessíveis e de alta qualidade antes e durante a gravidez, parto e período pós-natal. Também depende do apoio disponível para ajudar as mulheres grávidas a chegar aos serviços, principalmente quando ocorrem complicações. É plausível que os precursores das deficiências físicas das crianças possam ter sido evitados.

Deste estudo, cerca de um terço das mães lembrou que sua gravidez foi agitada. Para algumas das mães, patrocinando clínicas tradicionais para dar à luz, depois de um trabalho de parto prolongado e as coisas saíram do controle, elas vacilaram para os hospitais convencionais como alternativa. O patrocínio e a preferência por parteiras tradicionais (PTs) (no documento original é Traditional birth attendants(TBA) ainda são comuns na maioria dos países em desenvolvimento, como a Nigéria. Amutah-Onukagha e cols. ${ }^{23}$ relataram que, apesar dos avanços na saúde moderna, a PTs continuou a ser amplamente utilizada em comunidades rurais na Nigéria. Normalmente, os PTs são prestadores de serviços tradicionalmente independentes 
do sistema de saúde e prestadores de cuidados baseados na comunidade durante a gravidez, o parto e o período pósnatal. ${ }^{28}$ Os PTs não recebem treinamento médico formal quando comparados a outros profissionais de saúde, como obstetras e ginecologistas. No entanto, os PTs são muito mais baratos e acessíveis do que as parteiras qualificadas (SBAs) na maior parte do país. ${ }^{29}$ Devido à falta de educação em alguns $\mathrm{PTs}$, a forma como muitos compareceram ao parto é arriscada para as mulheres e seus bebês, levando a maus resultados de saúde e até mesmo morte. ${ }^{30}$

As consequências de gestações agitadas e complicações do parto por parte do bebê são deficiências, atrasos nos marcos e incapacidade. Especificamente, a partir deste estudo, mais da metade das crianças apresentou atraso no marco de desenvolvimento. $\mathrm{O}$ atraso no marco de desenvolvimento muitas vezes deixa os pais preocupados e iniciam a busca por cuidados. Estudos resumiram que, uma vez que surge uma preocupação com o desenvolvimento, muitos fatores afetam as respostas dos pais em busca de ajuda, incluindo qualidades pessoais (autoeficácia, conhecimento, habilidades, energia e persistência), influências e normas de pares e familiares e expectativas de que suas ações serão frutífero. ${ }^{31-33}$ Experiências anteriores de interações com os cuidados primários ou outros apoios formais (tratamento pela agência e sentimento de respeito pelo provedor) afetam fortemente a procura de ajuda e a tomada de decisão sobre o uso do serviço, assim como a confiança, as normas familiares e culturais, o estigma e a auto-estima práticas de cuidado.34,35

Esses motivos, além da incerteza quanto à saúde da criança, percepção de outras terapias e acesso aos cuidados identificados pelo presente estudo, desencadearam e facilitaram o itinerário terapêutico da criança com deficiência física. Conforme relatado em estudo anterior, é provável que os pais vejam a condição de seus filhos como uma emergência e estivessem em busca desesperada de uma solução. ${ }^{36}$ Não é improvável que a percepção de lares espirituais e tradicionalistas como oferendas de ajuda à saúde, desafios promovessem facilitação entre estes. prestadores de cuidados médicos ortodoxos. ${ }^{23}$ Também é notável que alguns cuidadores não ficaram satisfeitos com os cuidados ou receberam cuidados abaixo do ideal para facilitar a saúde e, portanto, os levaram à busca de melhores alternativas de cuidados". Pode ser que os profissionais de saúde que eles consultaram inicialmente sejam menos qualificados e, portanto, não poderiam fornecer o atendimento ideal, conforme sugerido por um estudo anterior. ${ }^{36}$

A partir do estudo atual, constatou-se que pais de crianças com deficiência física não se adaptam bem às condições de seus filhos. Em estudos anteriores, verificou-se que há uma variação considerável em como os pais se adaptam à demanda de cuidados. ${ }^{37,38}$ Alguns não se adaptam bem aos desafios de cuidar de uma criança com deficiência devido à consequência negativa representada pelo aumento da atenção física sobre eles. ${ }^{39,40} \mathrm{Em}$ países de baixa renda, muitos cuidadores de crianças com deficiência lutam com a pobreza, serviços públicos limitados e falta de dispositivos de assistência. Nessas situações, a prestação de cuidados pode exigir mais trabalho físico do que em países de alta renda e, portanto, acarretar maior risco de lesões físicas ou problemas de saúde. ${ }^{41}$ Assim, o papel da prestação de cuidados pode ser estressante e foi descoberto que influencia negativamente a saúde e bem-estar dos cuidadores..$^{40,42}$

Os achados desta pesquisa mostram que nos cuidadores cujos filhos apresentavam grau severo de deficiência física, o nível de dependência da criança dos demais membros da família é muito alto como observado anteriormente. ${ }^{10,43}$ Geralmente, mães de crianças com deficiência física têm pouco tempo para si, seja por causa das demandas de cuidados infantis ou porque optam por passar muito tempo com seu filho com deficiência física ${ }^{44}$ e descrevem consequências negativas para a saúde física, emocional e funcional de cuidados prolongados e informais. ${ }^{45}$ Essas observações estão de acordo com o presente estudo de que o itinerário terapêutico de cuidadores de crianças com deficiência física. tidade é um grande fardo para eles e muitas vezes impõe um fardo social e emocional para as crianças e suas famílias. ${ }^{46,47}$

As mães relataram o estigma financeiro e social como o retrocesso mais implicado no recebimento de cuidados em instalações hospitalares. Esse problema de baixa renda dos pais é ainda agravado pelo custo adicional de criar um filho com deficiência, ${ }^{48}$ bem como pela perda de redes sociais anteriores e do estigma. ${ }^{49} \mathrm{Em}$ países de baixa e média renda, fatores sociais e ambientais relacionados às situações em que as pessoas com deficiência e suas famílias vivem, influenciam as complexas interações entre deficiência, pobreza e saúde. ${ }^{22}$ Isso faz com que os cuidadores se envolvam em práticas de busca de cuidados múltiplos para seus filhos. ${ }^{50}$

É importante reconhecer que este foi um estudo com um grupo de crianças com deficiência em reabilitação em unidade de fisioterapia de um único hospital. A extensão em que as descobertas podem ter implicações mais amplas além desse cenário é uma das limitações do estudo.

\section{CONCLUSÃO E IMPLICAÇÕES PARA A PRÁTICA}

Ter filhos apresentando deficiências e marcos atrasados precipita práticas múltiplas de busca de cuidados de saúde complexos entre os cuidadores. Assim, os cuidadores tornam-se médicos pluralistas das práticas convencionais e tradicionais e isso pode favorecer o conflito ou a não adesão ao tratamento.

\section{CONTRIBUIÇÕES DOS AUTORES}

Concepção do desenho do estudo. Chidozie Emmanuel Mbada, Adetola Taiwo Onilude, Olufemi Oyeleye Oyewole, Oluwatosin Eunice Olorunmoteni, Clara Fatoye, Abiola Oladele Ogundele, Francis Fatoye.

Aquisição de dados. Chidozie Emmanuel Mbada, Adetola Taiwo Onilude, Olufemi Oyeleye Oyewole, Oluwatosin Eunice Olorunmoteni, Clara Fatoye, Abiola Oladele Ogundele, Francis Fatoye. 
Análise de dados. Chidozie Emmanuel Mbada, Adetola Taiwo Onilude, Olufemi Oyeleye Oyewole, Oluwatosin Eunice Olorunmoteni, Clara Fatoye, Abiola Oladele Ogundele, Francis Fatoye.

Interpretação dos achados. Chidozie Emmanuel Mbada, Adetola Taiwo Onilude, Olufemi Oyeleye Oyewole, Oluwatosin Eunice Olorunmoteni, Clara Fatoye, Abiola Oladele Ogundele, Francis Fatoye.

Escrita e revisão crítica do manuscrito. Chidozie Emmanuel Mbada, Adetola Taiwo Onilude, Olufemi Oyeleye Oyewole, Oluwatosin Eunice Olorunmoteni, Clara Fatoye, Abiola Oladele Ogundele, Francis Fatoye.

Aprovação da versão final do manuscrito. Chidozie Emmanuel Mbada, Adetola Taiwo Onilude, Olufemi Oyeleye Oyewole, Oluwatosin Eunice Olorunmoteni, Clara Fatoye, Abiola Oladele Ogundele, Francis Fatoye.

Responsabilizar-se por todos os aspectos e conteúdos do manuscrito, a acurária e integridade científica do artigo publicado. Chidozie Emmanuel Mbada, Adetola Taiwo Onilude, Olufemi Oyeleye Oyewole, Oluwatosin Eunice Olorunmoteni, Clara Fatoye, Abiola Oladele Ogundele, Francis Fatoye.

\section{EDITOR ASSOCIADO}

Franco Carnevale (1)

\section{EDITOR CIENTÍFICO}

Ivone Evangelista Cabral (1)

\section{REFERÊNCIAS}

1. World Health Organization. The global burden of disease: 2004 update Geneva: WHO; 2008. [citado 2020 abr 9]. Disponível em: https://www. who.int/healthinfo/global_burden_disease/2004_report_update/en/

2. World Health Organization. World report on disability 2011. Geneva: WHO; 2011. [citado 2020 abr 9]. Disponível em: https://apps.who.int/ iris/handle/10665/44575

3. Kandel I, Merrick J. The Birth of a Child with Disability. Coping by parents and siblings. ScientificWorldJournal. 2003;3:741-50. http:// dx.doi.org/10.1100/tsw.2003.63. PMid:12953123.

4. Brown I, Brown RI. Quality of life and disability: an approach for community practitioners. 1st ed. London: Jessica Kingsley; 2003.

5. Hastings RP, Johnson E. Stress in UK families conducting intensive homebased behavioral intervention for their young child with autism. J Autism Dev Disord. 2001;31(3):327-36. http://dx.doi.org/10.1023/A:1010799320795. PMid: 11518485

6. Jones J, Passey J. Family Adaptation, Coping and Resources: Parents Of Children With Developmental Disabilities and Behaviour Problems. J Dev Disabil. 2004;11(1):31-46.

7. Nurullah AS. "It's Really a Roller Coaster": Experience of Parenting Children with Developmental Disabilities. Marriage Fam Rev. 2013;49(5):412-45. http://dx.doi.org/10.1080/01494929.2013.768320.

8. Green SE. "What do you mean 'what's wrong with her?"': stigma and the lives of families of children with disabilities. Soc Sci Med. 2003;57(8):13611374. http://dx.doi.org/10.1016/s0277-9536(02)00511-7.

9. Cadman D, Rosenbaum P, Boyle M, Offord DR. Children with chronic illness: family and parent demographic characteristics and psychosocial adjustment. Pediatrics. 1991;87(6):884-9. PMid:2034494.

10. Schuengel C, Rentinck ICM, Stolk J, Voorman JM, Loots GMP, Ketelaar $M$ et al. Parents' reactions to the diagnosis of cerebral palsy: associations between resolution, age and severity of disability. Child Care Health Dev 2009;35(5):673-80. http://dx.doi.org/10.1111/j.1365-2214.2009.00951.x. PMid:19320906.

11. Plant KM, Sanders MR. Predictors of care-giver stress in families of preschool-aged children with developmental disabilities. J Intellect Disabil Res JIDR. 2007;51(Pt2):109-24. http://dx.doi.org/10.1111/j.13652788.2006.00829.x. PMid:17217475.

12. McCarthy A, Cuskelly M, van Kraayenoord CE, Cohen J. Predictors of stress in mothers and fathers of children with fragile $X$ syndrome. Res Dev Disabil. 2006;27(6):688-704. http://dx.doi.org/10.1016/j. ridd.2005.10.002. PMid:16361079.

13. Neely-Barnes S, Marcenko M. Predicting impact of childhood disability on families: results from the 1995 National Health Interview Survey Disability Supplement. Ment Retard. 2004;42(4):284-93. http://dx.doi. org/10.1352/0047-6765(2004)42<284:PIOCDO>2.0.CO;2. PMid:15230650.

14. Verma RK, Kishore MT. Needs of Indian parents having children with intellectual disability. Int J Rehabil Res. 2009;32(1):71-6. http://dx.doi. org/10.1097/MRR.0b013e32830d36b6. PMid:19648804.

15. Gerhardt S. Why love matters: How affection shapes a baby's brain. Infant Obs. 2006;9(3):305-9. http://dx.doi.org/10.1080/13698030601074476.

16. Beresford B. Expert Opinions: A national survey of parents caring for a severely disabled child [Internet]. Bristol: Policy Press; 1995. [citado 2020 abr 9]. Disponível em: https://pure.york.ac.uk/portal/en/publications/ expert-opinions-a-national-survey-of-parents-caring-for-a-severelydisabled-child(0a5a2a0c-068b-407f-962f-209b18b0c6e3)/export.htm

17. Whiting M. Impact, meaning and need for help and support: The experience of parents caring for children with disabilities, life-limiting/ life-threatening illness or technology dependence. J Child Health Care. 2013;17(1):92-108. http://dx.doi.org/10.1177/1367493512447089. PMid:23220736.

18. Sloper T, Beresford B. Families with disabled children. BMJ. 2006;333(7575):928-9. http://dx.doi.org/10.1136/bmj.39017.633310. BE. PMid:17082523.

19. Hewitt-Taylor J. Providing support at home for families whose children have complex needs. J Child Young Peoples Nurs. 2007;1(4):195-200. http://dx.doi.org/10.12968/jcyn.2007.1.4.24399.

20. Shewchuk RM, Richards JS, Elliott TR. Dynamic processes in health outcomes among caregivers of patients with spinal cord injuries. Health Psychol Off J Div Health Psychol Am Psychol Assoc. 1998;17(2):125-9. http://dx.doi.org/10.1037/0278-6133.17.2.125. PMid:9548703.

21. Frank-Briggs AI, D Alikor EA. Pattern of paediatric neurological disorders in Port Harcourt, Nigeria. Int J Biomed Sci. 2011;7(2):145-9. PMid:23675231.

22. Parnes P, Cameron D, Christie N, Cockburn L, Hashemi G, Yoshida K Disability in low-income countries: Issues and implications. Disabil Rehabil. 2009;31(14):1170-80. http://dx.doi.org/10.1080/09638280902773778. PMid:19802932.

23. Amutah-Onukagha N, Rodriguez M, Opara I, Gardner M, Assan MA, Hammond $R$ et al. Progresses and challenges of utilizing traditional birth attendants in maternal and child health in Nigeria. Int J MCH AIDS. 2017;6(2):130-8. http://dx.doi.org/10.21106/ijma.216. PMid:29367889.

24. Creswell JW. Qualitative inquiry and research design: Choosing among five traditions. Thousand Oaks, CA: Sage Publications, Inc; 1998.

25. Braun V, Clarke V, Hayfield N, Terry G. Thematic Analysis. In: Liamputtong $\mathrm{P}$, editor. Handbook of research methods in health social sciences. Singapore:Springer;2019. p. 843-860. doi:http://dx.doi.org/10.1007/978981-10-5251-4_103

26. Kling A, Campbell PH, Wilcox J. Young children with physical disabilities: Caregiver perspectives about assistive technology. Infants Young Child 2010;23(3):169-83. http://dx.doi.org/10.1097//YC.0b013e3181e1a873.

27. Wallace AG. Maternal health in Uganda: Understanding the low utilization of skilled attendants in Mukono, Uganda through women's experiences in the health care system [thesis]. Halifax, Nova Scotia: Saint Mary's University; 2012 [citado 2020 abr 9]. Disponível em: http://library2.smu. $\mathrm{ca} / \mathrm{xmlui} /$ handle/01/25210

28. World Health Organization. The Partnership for Maternal, Newborn and Child Health 2016 annual report: coming of age in a time of transition 
[Internet]. Geneva: World Health Organization; 2017 [citado 2020 abr 9]. Disponível em: https://apps.who.int/iris/handle/10665/258801

29. Balogun M, Odeyemi K. Knowledge and practice of prevention of mother-to-child transmission of HIV among traditional birth attendants in Lagos State, Nigeria. Pan Afr Med J. 2010;5:7. PMid:21120006.

30. Mrisho M, Schellenberg JA, Mushi AK, Obrist B, Mshinda H, Tanner $M$ et al. Factors affecting home delivery in rural Tanzania. Trop Med Int Health TM IH. 2007;12(7):862-72. http://dx.doi.org/10.1111/j.13653156.2007.01855.x. PMid:17596254.

31. Marshall J. Parent Pathways: Recognition and responses to developmental delays in young children [dissertation]. Tampa, FL: University of South Florida, 2013.

32. Toran $\mathrm{H}$. Experience and challenges in setting up a model demonstration classroom for children with autism in Malaysia. Int J Educ Adm Dev. 2011;2(2):37-47.

33. Turnbull AP, Turnbull HR. From the old to the new paradigm of disability and families: Research to enhance family quality of life outcomes. In: Paul J, Lavely, CD, Cranston-Gingras, A, editor. Professional issues in special education: Intellectual, ethical, and professional challenges to the profession. Westport, CT: Greenwood Publishing Group, Inc. Ablex Publishing; 2002. p. 83-119.

34. Gance-Cleveland B. Family-centered care. Decreasing health disparities. J Spec Pediatr Nurs JSPN. 2006;11(1):72-6. http://dx.doi. org/10.1111/j.1744-6155.2006.00046.x. PMid:16409509.

35. Kerkorian D, McKay M, Bannon Jr WM. Seeking help a second time: parents'/caregivers' characterizations of previous experiences with mental health services for their children and perceptions of barriers to future use. Am J Orthopsychiatry. 2006;76(2):161-6. http://dx.doi. org/10.1037/0002-9432.76.2.161. PMid:16719634.

36. Luz R, Pieszak G, Arrue A, Gomes G, Neves E, Rodrigues A. Therapeutic itinerary of families of children with special health needs. Rev Rede Enferm Nordeste. 2019;20:e33937. http://dx.doi.org/10.15253/21756783.20192033937.

37. Sloper P. Models of service support for parents of disabled children. What do we know? What do we need to know? Child Care Health Dev. 1999;25(2):85-99. http://dx.doi.org/10.1046/j.1365-2214.1999.25220120.x. PMid:10188064.

38. Wallander JL, Varni JW. Effects of pediatric chronic physical disorders on child and family adjustment. J Child Psychol Psychiatry. 1998;39(1):2946. http://dx.doi.org/10.1017/S0021963097001741. PMid:9534085.

39. Raina P, O'Donnell M, Rosenbaum P, Brehaut J, Walter SD, Russell $D$ et al. The health and well-being of caregivers of children with cerebral palsy. Pediatrics. 2005;115(6):e626-36. http://dx.doi.org/10.1542/ peds.2004-1689. PMid:15930188.

40. Davies RC. The playing robot: Helping children with disabilities to play IFAC Proc Vol. 1995;28(20):63-68. http://dx.doi.org/10.1016/S14746670(17)45026-9

41. Geere JL, Gona J, Omondi FO, Kifalu MK, Newton CR, Hartley S. Caring for children with physical disability in Kenya: potential links between caregiving and carers' physical health. Child Care Health Dev. 2013;39(3):381-92. http://dx.doi.org/10.1111/j.1365-2214.2012.01398.x. PMid:22823515.

42. Quittner AL, Glueckauf RL, Jackson DN. Chronic parenting stress: moderating versus mediating effects of social support. J Pers Soc Psychol. 1990;59(6):1266-78. http://dx.doi.org/10.1037/0022-3514.59.6.1266. PMid:2283593.

43. Lima M, Silva K, Amaral I, Magalhães A, de Sousa L. Beyond behavioura observations: a deeper view through the sensory reactions of children with profound intellectual and multiple disabilities. Child Care Health Dev. 2013;39(3):422-31. http://dx.doi.org/10.1111/j.1365-2214.2011.01334.x. PMid:22017606.

44. Hill EJ, Hawkins AJ, Märtinson V, Ferris M. Studying "Working Fathers": Comparing Fathers' and Mothers' Work-Family Conflict, Fit, and Adaptive Strategies in a Global High-Tech Company. Father J Theory Res Pract Men Fathers. 2003;1(3):239-61. http://dx.doi.org/10.3149/fth.0103.239.

45. Murphy NA, Christian B, Caplin DA, Young PC. The health of caregivers for children with disabilities: caregiver perspectives. Child Care Health Dev. 2007;33(2):180-7. http://dx.doi.org/10.1111/j.1365-2214.2006.00644.x. PMid:17291322.

46. Ryan SE, Campbell KA, Rigby PJ. Reliability of the family impact of assistive technology scale for families of young children with cerebra palsy. Arch Phys Med Rehabil. 2007;88(11):1436-40. http://dx.doi. org/10.1016/j.apmr.2007.06.777. PMid:17964884.

47. Farmer JE, Marien WE, Clark MJ, Sherman A, Selva TJ. Primary care supports for children with chronic health conditions: identifying and predicting unmet family needs. J Pediatr Psychol. 2004;29(5):355-67. http://dx.doi.org/10.1093/jpepsy/jsh039. PMid:15187174.

48. Lukemeyer A, Meyers MK, Smeeding T. Expensive Children in Poor Families: Out-of-Pocket Expenditures for the Care of Disabled and Chronically III Children in Welfare Families. J Marriage Fam. 2000;62(2):399-415. http://dx.doi.org/10.1111/j.1741-3737.2000.00399.x.

49. Bain KJ. Children with severe disabilities: options for residential care. Med J Aust. 1998;169(11-12):598-600. http://dx.doi.org/10.5694/j.1326-5377.1998. tb123430.x. PMid:9887905.

50. Simms R. Looking abroad for treatment. Pac IsI Mon. 1992;62(10):34-5. 